\title{
Reflets
}

Revue d'intervention sociale et communautaire

\section{Le potentiel d'offre et de demande de services médicaux dans la langue de communautés minoritaires : où se situe le français en Colombie-Britannique?}

\section{Louis Giguère et Brian Conway}

Volume 20, numéro 2, automne 2014

L'accès aux services sociaux et de santé en français et la formation des professionnelles et professionnels en situation francophone minoritaire canadienne

URI : https://id.erudit.org/iderudit/1027586ar

DOI : https://doi.org/10.7202/1027586ar

Aller au sommaire du numéro

Éditeur(s)

Reflets, Revue d'intervention sociale et communautaire

ISSN

1203-4576 (imprimé)

1712-8498 (numérique)

Découvrir la revue

Citer cet article

Giguère, L. \& Conway, B. (2014). Le potentiel d'offre et de demande de services médicaux dans la langue de communautés minoritaires : où se situe le français en Colombie-Britannique? Reflets, 20(2), 52-82.

https://doi.org/10.7202/1027586ar
Résumé de l'article

Une étude comparative du potentiel de services médicaux dans les langues minoritaires de Colombie-Britannique révèle que des médecins non-francophones parlant français génèrent une retombée linguistique qui amplifie l'offre potentielle de services de santé et de services sociaux en français de façon dramatique. Ce mécanisme structurant s'appliquerait aux autres professions de la santé et des services sociaux, partout au Canada et il serait plus prononcé plus le contexte est minoritaire. Les communautés francophones doivent faire plus pour identifier, engager et outiller les professionnels et professionnelles « francophiles » et elles doivent élaborer une logique d'équité innovatrice pour dégager le potentiel d'offre de services de santé et de services sociaux en français dans un contexte multilingue.
Tous droits réservés @ Reflets, Revue d’intervention sociale et communautaire, 2014
Ce document est protégé par la loi sur le droit d'auteur. L'utilisation des services d'Érudit (y compris la reproduction) est assujettie à sa politique d'utilisation que vous pouvez consulter en ligne. 


\title{
Le potentiel d'offre et de demande de services médicaux dans la langue de communautés minoritaires : où se situe le français en Colombie-Britannique?
}

\author{
Louis Giguère \\ RésoSanté Colombie-Britannique (RSCB) \\ Brian Conway \\ RésoSanté Colombie-Britannique (RSCB)
}

\section{Résumé}

Une étude comparative du potentiel de services médicaux dans les langues minoritaires de Colombie-Britannique révèle que des médecins non-francophones parlant français génèrent une retombée linguistique qui amplifie l'offre potentielle de services de santé et de services sociaux en français de façon dramatique. Ce mécanisme structurant s'appliquerait aux autres professions de la santé et des services sociaux, partout au Canada et il serait plus prononcé plus le contexte est minoritaire. Les communautés francophones doivent faire plus pour identifier, engager et outiller les professionnels et professionnelles " francophiles " et elles doivent élaborer une logique d'équité innovatrice pour dégager le potentiel d'offre de services de santé et de services sociaux en français dans un contexte multilingue. 
Mots clés : CFASM, CLOSM, médecins parlant français, minorités linguistiques, équité, services d'interprétation, offre et demande

\section{Abstract}

A comparative study of medical services potential in linguistic minorities languages in British Columbia reveals that nonFrancophones French-speaking doctors create a linguistic spinoff that dramatically boosts the potential supply of health and social services in French. This structuring mechanism would apply to other health and social services, elsewhere in Canada and it would be stronger the greater the minority context. Francophone communities must do more to identify, engage and empower francophile professionals and they must develop an innovative equity rationale to unleash the potential for health and human services in French in a multilingual context.

Key words: FACMS, OLMC, French-speaking doctors, linguistic minorities, equity, interpretation services, offer and demand

\section{Introduction}

Pour favoriser les services gouvernementaux dans la langue de choix de ses minorités de langues officielles le Canada a adopté des lois qui obligent les institutions fédérales à favoriser le développement et l'épanouissement de ces communautés (Ministère de la justice, 1985; 2005) (Patrimoine canadien, 2007). Côté santé, Santé Canada a adopté une stratégie de services de santé en français souple et différentielle (CCCFSM, 2001;2007) ancrée dans 17 réseaux santé communautaires pour mieux composer avec les situations diverses qui caractérisent les Communautés Francophones et Acadiennes en Situation Minoritaire (CFASM) du Canada.

Pour sa part, le réseau de Colombie-Britannique, RésoSanté, œuvre dans une CFASM assez grande ( $4^{\mathrm{e}}$ en termes de population) 
"Les francophones ont un poids démographique faible dans cette province $(1,7 \%$ au recensement de 2006 et $1,5 \%$ en 2011), sont relativement dispersés et grandement assimilés ...comme la Colombie-Britannique comprend 29,4\% d'immigrants de première génération (National Post, 2013), le français ne vient qu'au $10^{e}$ rang en termes du nombre de locuteurs de langues minoritaires parlées à la maison.» mais plutôt fragile. Les francophones ont un poids démographique faible dans cette province $(1,7 \%$ au recensement de 2006 et 1,5\% en 2011), sont relativement dispersés et grandement assimilés (FCFA, 2007) (Chavez et Bouchard-Coulombe, 2011). De plus, comme la Colombie-Britannique comprend 29,4 \% d'immigrants de première génération (National Post, 2013), le français ne vient qu'au $10^{\mathrm{e}}$ rang en termes du nombre de locuteurs de langues minoritaires parlées à la maison ${ }^{1}$. Comme il y a plusieurs minorités plus importantes que le français en termes de poids démographique et que certaines sont relativement grandes, il y est difficile de justifier une augmentation de l'offre de services de santé en français.

Trois études de besoin ont établi qu'il est difficile d'obtenir des services de santé en français en Colombie-Britannique, soit RésoSanté $(2005$, p. 60) «Au cours des trois dernières années, $41 \%$ des répondants ont tenté de consulter un professionnel de la santé parlant le français en Colombie-Britannique. Or la majorité d'entre eux (58,3 \%) n'ont pas reçu de service en français ", RésoSanté (2006, p. 2) « Parmi ceux qui ont essayé de trouver un médecin francophone, $73 \%$ ont eu de la difficulté et $52 \%$ n'en n'ont pas trouvé ", et Beaulieu (2010,p. 37) "Comme ailleurs au pays, leur plus grand défi consiste à trouver des professionnels qui peuvent s'exprimer dans les deux langues officielles ».

De plus, trois études issues de l'Enquête sur la vitalité des minorités de langues officielles du recensement de 2006 de Statistique Canada ont confirmé que les niveaux de services en français ne répondent pas aux attentes des francophones (Gagnon-Arpin, 2011) (Giguère, 2013; 2014).

Pour pallier à cette pénurie de services RésoSanté s'est appuyé il y a dix ans sur le « Fonds d'adaptation des soins de santé primaires » de Santé Canada pour collaborer avec une régie de santé, Vancouver Coastal Health Authority, (VCHA) afin de mettre en œuvre des initiatives de services de santé en français, dont une clinique sur le diabète, une clinique sur l'autisme, un modèle d'accès à des services en français pour les démunis francophones et une page d'accès Web pour desservir l'ensemble de la population francophone. En dedans de quatre années cependant, ces quatre 
"...l'interprétation est l'approche la moins prisée parmi celles proposées aux francophones selon l'étude de besoins "Préparer le terrain en ColombieBritannique " menée par RésoSanté. » initiatives ont été abandonnées, dont une en raison du fait que ces services étaient perçus comme non-équitables par rapport à d'autres minorités linguistiques de poids démographique plus considérable. Concomitamment on observait que le système de santé s'investissait de plus en plus dans une approche axée sur l'interprétation, une approche qui semble lui permettre de composer plus facilement avec le défi de fournir des services à une population multilingue et multiculturelle grandissante (Gabriel, 2012). La régie provinciale de santé de Colombie-Britannique a expérimenté avec cette approche pendant plusieurs années et a donné fruit à un service d'interprétation de qualité qui opère depuis une dizaine d'années dans 250 langues, 24 heures par jour. Ce service, le Provincial Language Service (PLS), a récemment conclu des ententes de service avec les régies de santé VCHA et Fraser Health ainsi qu'avec l'agence Providence Health Care pour répondre à leurs besoins d'interprétation médicale dans la grande région de Vancouver. Plus récemment, une initiative d'interprétation voyait le jour dans un milieu de cliniques de médecine familiale du Grand Vancouver Est (Gabriel, 2012).

Cependant l'interprétation est l'approche la moins prisée parmi celles proposées aux francophones selon l'étude de besoins "Préparer le terrain en Colombie-Britannique " menée par RésoSanté. Ces derniers se disent mal à l'aise de partager leurs problèmes de santé avec des tierces parties (RésoSanté, 2006, p. 24 et 58) et ce malaise se reflète dans les statistiques d'utilisation des services d'interprétation du PLS pour 20012-2013 et 20132014 : bien que les francophones représentent de 1,5 à 1,7 \% de la population de la province, le français représente seulement $0,4 \%$ de la demande de services d'interprétation (soit 4 fois moins que le poids démographique, PLS 2014). L'approche d'interprétation est donc considérée comme complémentaire plutôt que prioritaire par RésoSanté.

Pour mieux situer les défis de l'offre active de services en français dans le contexte multilingue et multiculturel de Colombie-Britannique, nous nous sommes fixés deux objectifs de recherche : 
1- Comparer le potentiel d'offre et de demande de services médicaux (soit le nombre de médecins parlant une langue et le poids démographique d'une population de langue maternelle, respectivement) pour les minorités linguistiques de Colombie-Britannique et dresser un portrait d'ensemble pour mieux comprendre les enjeux; et

2- Comparer et contraster le français par rapport à ce portrait d'ensemble pour essayer d'identifier des caractéristiques structurantes uniques au français.

Nous viserons en discussion à positionner la communauté francophone de façon singulière et à proposer des pistes d'amélioration des services en français qui soient équitables dans le contexte multilingue et multiculturel de la Colombie-Britannique.

\section{Méthodes}

Nous avons calculé les indicateurs suivants pour 97 langues qui sont identifiées au site Web du "BC College of Physicians and Surgeons" (BCPS) et aux recensements canadiens de 2006 et 2011. Nous mettons ici l'accent sur les 30 plus grandes communautés linguistiques pour simplifier la présentation.

\section{Un indicateur d'offre de services pour une langue}

"Le nombre de médecins parlant une langue est un indicateur $d u$ potentiel d'offre pour la communauté parlant cette langue. »
Le nombre de médecins parlant une langue est un indicateur du potentiel d'offre pour la communauté parlant cette langue $\left(\mathrm{I}_{1}\right)$. Nous avons choisi cette statistique parce qu'elle est disponible sur le site Web du "British Columbia College of Physicians and Surgeons" (BCCPS) depuis 2008 et parce l'accès à un médecin est considéré comme prioritaire par les francophones de Colombie-Britannique (RésoSanté, 2005; 2006). Le BCCPS met ces données à jour annuellement, en début d'année. Nous avons retiré des données pour chacune des langues parlées par les médecins pratiquants pour cinq années soit le 4 mai 2009, le 16 avril 2010, le 15 juillet 2011, le 7 mai 2012 et le 5 février 2014. Ces médecins ont auto-évalué leur capacité langagière mais nous les décrivons comme étant des "professionnels ou professionnelles parlant pendjabi », etc. 


\section{Deux indicateurs de demande de services pour une langue}

Le premier indicateur $\left(\mathrm{I}_{2}\right)$ reflète le potentiel maximum de demande, soit la population de langue maternelle. Ces données probantes du recensement national de 2011 sont disponible sur le site Web de Statistique Canada (tableau populationnel 98-314-X2011016 sur la langue maternelle). Ce résultat de poids démographique permet d'estimer la demande si tous et chacun demandaient des services dans leur langue maternelle. Comme les membres d'une minorité linguistique peuvent demander des services dans la langue de la majorité, la demande réelle sera moindre.

Le deuxième indicateur $\left(\mathrm{I}_{3}\right)$ reflète un potentiel pondéré, soit la population qui parle le plus souvent sa langue maternelle à la maison. Ces données probantes sont tirées du tableau 98314-X2011042 du recensement national de 2011 de Statistique Canada. Cet indicateur pondéré est important d'une perspective comparative parce que la langue parlée à la maison reflète la vitalité linguistique d'une communauté (et inversement son assimilation), selon plusieurs études nationales (Chartrand, 2010) (Giguère 2013) (IRFA, 2012) (Lacroix et Sabourin, 2006) (Landry, 2010) (Lentz, 2004) et internationales (Aleksynska et Algan, 2010) (Chiswick, 1991) (Dustmann, 1994). Une communauté linguistique plus assimilée est moins susceptible de demander des services dans sa langue maternelle.

\section{Un indicateur du niveau d'offre de services pour une langue}

Cet indicateur représente le nombre de médecins par 1000 de capita d'une communauté linguistique $\left(\mathrm{I}_{3}\right)$ et est obtenu à partir de deux des indicateurs précédents en multipliant le nombre de médecins parlant une langue $\left(\mathrm{I}_{1}\right)$ par 1000 et en divisant ce résultat par la population de langue maternelle correspondante $\left(\mathrm{I}_{2}\right)$. Cet indicateur est une mesure relative qui permet de comparer le niveau de services d'une langue à l'autre (Warnke, 2009) (Warnke et Bouchard, 2013), mais on doit l'utiliser prudemment pour faire des comparaisons avec des indicateurs de mesure absolue pour l'ensemble d'une juridiction provinciale, territoriale, nationale ou internationale. Par exemple l'indicateur décompte un médecin qui 
parle plusieurs langues plusieurs fois (ce biais n'est pas considérable cependant). Le potentiel de services ne pourrait être réalisé pour une langue que s'il y avait un appariement parfait entre les médecins et leur clientèle, selon la langue maternelle du client. En réalité il y a un biais d'appariement.

\section{Un indicateur d'offre de services en français}

"Comme nos résultats suggéraient en cours de route que beaucoup de médecins parlant français en ColombieBritannique sont des Anglophones ou des Allophones ... nous avons dressé un portrait sommaire de la source d'apprentissage du français pour mieux comprendre les enjeux."
Comme nos résultats suggéraient en cours de route que beaucoup de médecins parlant français en Colombie-Britannique sont des Anglophones ou des Allophones (que nous désignerons "médecins francophiles » à toutes fins pratiques), nous avons dressé un portrait sommaire de la source d'apprentissage du français pour mieux comprendre les enjeux. Comme nous voulions que notre échantillon représente un portrait de l'offre récent, nous avons limité la population cible aux médecins de pratique générale ayant gradué depuis l'an 2000 selon le site Web du BCCPS. Nous avons suivi un ordre alphabétique pour les 15 premiers choix et un ordre systématique pour les 35 suivants $\left(l e 1^{\text {er }}\right.$, le $5^{\text {e }}$, le $9^{\text {e }}$ médecin etc., une méthode stratifiée au hasard; Zar, 1984). Nous avons déterminé en février et en juin 2014, par conversation téléphonique, combien étaient francophones de souche, combien avaient appris le français comme langue seconde en vivant dans une juridiction où on parle français, ou en apprentissage langue seconde dans une école au Canada (programme de base ou programme d'immersion française). Certaines de ces catégories ne sont pas strictement mutuellement exclusives (par exemple un élève peut faire une partie de sa formation scolaire en programme de base et une autre partie en immersion française, ou un médecin qui vit à Montréal peut aussi avoir participé à un programme d'immersion française au Québec) mais adéquates pour un portrait d'ensemble. Nous avons réussi à obtenir une réponse pour 38 des 164 médecins ciblés, soit 23,2\%.

\section{Autres considérations méthodologiques}

Comme Warnke et Bouchard (2013) nous avons choisi la statistique populationnelle de réponse unique pour identifier la langue 
parlée. Une définition combinant les réponses unique et multiples est souvent utilisée (la définition PLOP ou première langue officielle parlée (Chavez et Bouchard-Coulombe, 2011) (Forgues et Landry, 2006) (Forgues, Landry et Boudreau, 2009) (Pocock et collab., 2011). À notre avis la mesure PLOP ne serait pas fiable sur un ensemble de communautés linguistiques très diverses.

\section{Résultats et interprétation}

\section{A- Comparaison du potentiel d'offre et de demande de services médicaux pour les plus grandes minorités linguistiques de Colombie-Britannique : Portrait d'ensemble}

(a) Le potentiel d'offre de services médicaux $\left(I_{1}\right)$ soit le nombre de médecins parlant une langue minoritaire, 2009 à 2014 (communautés $>10000$ de population, langue maternelle)

Les résultats bruts sont présentés en Annexe 1. De loin, le français vient au premier rang du potentiel d'offre, soit 1161 médecins en 2014. On observe trois patrons de croissance.

Pour dix communautés (chinois n.d.a., allemand, français, espagnol, persan, italien, néerlandais, japonais, polonais, portugais) la croissance annuelle est de $1,7 \%$ ce qui correspond d'assez près à un taux de croissance annuel de $1,9 \%$ sur l'ensemble des médecins pour la même période. On observe aussi que le nombre de médecins baisse les deux premières années puis remonte en 2014. Nous attribuons cet effet à un changement au questionnaire du BCCPS. En 2010, 2011 et 2012 on demandait aux médecins dans quelle(s) langue(s) ils pouvaient exercer leur pratique professionnelle, à part l'anglais, alors qu'en 2009 et 2014 on leur demandait quelle(s) autre(s) langue(s) ils pouvaient parler, à part l'anglais. Lorsque la question du BCCPS porte sur la compétence en pratique professionnelle plutôt que sur la connaissance d'une langue, le nombre de réponses positives baisse de $36,2 \%$, en dépit d'un taux annuel de croissance de 1,9\%. 
"...pour la plupart

des minorités

linguistiques de

Colombie-Britannique, le potentiel d'offre

de services médicaux

a une croissance

faible ....alors que

pour les trois plus

grandes communautés

linguistiques, il

augmente très

rapidement... »
Une baisse d'approximativement un tiers représente donc un barème de la compétence en pratique professionnelle par rapport à la compétence langagière, pour cette profession et pour cette juridiction.

Pour cinq communautés dont le nombre de médecins est petit $(<90)$, on n'observe pas de croissance (tagalog, coréen, vietnamien, russe, arabe). Finalement, pour quatre communautés dont les trois premières sont les plus grandes minorités de Colombie-Britannique (pendjabi, cantonnais, mandarin, hindi), on observe une croissance annuelle du nombre de médecins de 19,1\%. Cette croissance dépasse largement le taux de croissance annuel de médecins $(1,9 \%)$ et le taux de croissance annuel pour ces communautés (4,4 \%-pendjabi, 1,2\%-cantonnais, 6,7\%-mandarin, et 4,2\%-hindi, selon les recensements de 2006 et de 2011).

Nous concluons donc que pour la plupart des minorités linguistiques de Colombie-Britannique, le potentiel d'offre de services médicaux a une croissance faible (soit qu'il soit statique ou qu'il soit en proportion avec le taux de croissance global du nombre de médecins en Colombie-Britannique) alors que pour les trois plus grandes communautés linguistiques, il augmente très rapidement (10x plus que pour les autres communautés). Une telle croissance ne semble possible que s'il existe un effort de recrutement soutenu pour faire augmenter l'offre de services pour ces communautés.

b) Le potentiel de demande de services médicaux $\left(I_{2}\right.$ et $\left.I_{3}\right)$ soit les populations par langue maternelle et par langue parlée à la maison, 2011 (communautés $>5000$ de population, langue maternelle)

Pour cette section et la prochaine section nous avons choisi les données de 2011 parce qu'elles sont basées sur la compétence en pratique professionnelle et parce qu'elles coïncident avec une année de recensement.

Le rang par population de langue maternelle est indiqué en colonne 1 (Tableau 1). On observe 3 communautés asiatiques au- 
dessus de 100000 de population, 4 communautés entre 50000 et 100000 de population et 3 communautés entre 20000 et 50000 de population. Le français vient au $7^{\mathrm{e}} \mathrm{rang}$ du potentiel de la demande avec une population de 57280.

Tableau 1 - Quatre indicateurs linguistiques pour 30 langues communément parlées en Colombie-Britannique en 2011.

\begin{tabular}{|c|c|c|c|c|c|}
\hline Rang* & Langue & $\begin{array}{c}\mathrm{I}_{1} \\
\text { No. de } \\
\text { médecins }\end{array}$ & $\begin{array}{c}\mathrm{I}_{2} \\
\text { Population, } \\
\text { langue } \\
\text { maternelle }\end{array}$ & $\begin{array}{c}\text { I }_{3} \\
\text { Population, langue } \\
\text { parlée à la maison }\end{array}$ & $\begin{array}{c}\mathrm{I}_{4} \\
\text { No. de médecins/ } \\
1000 \text { de } \\
\text { population }\end{array}$ \\
\hline 1 & Pendjabi & 249 & 182920 & 137390 & 1,4 \\
\hline 2 & Cantonnais & 370 & 133245 & 103550 & 2,8 \\
\hline 3 & Chinois n.d.a. & 183 & 120040 & 81270 & 1,5 \\
\hline 4 & Mandarin & 207 & 94055 & 78075 & 2,2 \\
\hline 5 & Allemand & 169 & 73625 & 11550 & 2,3 \\
\hline 6 & Tagalog & 17 & 66120 & 29380 & 0,3 \\
\hline 7 & Français & 683 & 57280 & 16685 & 11,9 \\
\hline 8 & Coréen & 21 & 49975 & 38810 & 0,4 \\
\hline 9 & Espagnol & 148 & 40790 & 20585 & 3,6 \\
\hline 10 & Persan & 18 & 36045 & 24750 & 0,5 \\
\hline 11 & Vietnamien & 24 & 25105 & 17270 & 1,0 \\
\hline 12 & Hindi & 240 & 24640 & 12390 & 9,7 \\
\hline 13 & Italien & 45 & 24060 & 6250 & 1,9 \\
\hline 14 & Néerlandais & 48 & 23080 & 2230 & 2,1 \\
\hline 15 & Russe & 60 & 22650 & 12200 & 2,6 \\
\hline 16 & Japonais & 9 & 19665 & 9540 & 0,5 \\
\hline 17 & \begin{tabular}{|l} 
Polonais \\
\end{tabular} & 66 & 17455 & 6395 & 3,8 \\
\hline 18 & Portugais & 15 & 14610 & 5190 & 1,0 \\
\hline 19 & Arabe & 82 & 13050 & 7635 & 6,3 \\
\hline 20 & Ukrainien & 22 & 9680 & 1070 & 2,3 \\
\hline 21 & Hongrois & 23 & 9680 & 2565 & 2,4 \\
\hline 22 & Ourdou & 133 & 8450 & 5425 & 15,7 \\
\hline
\end{tabular}




\begin{tabular}{|c|l|c|c|c|c|}
\hline 23 & Roumain & 27 & 8330 & 4155 & 3,2 \\
\hline 24 & Croate & 16 & 7820 & 2540 & 2,0 \\
\hline 25 & Gujarati & 65 & 6590 & 3045 & 9,9 \\
\hline 26 & Serbe & 18 & 6655 & 4015 & 2,7 \\
\hline 27 & Grec & 14 & 6250 & 2105 & 2,2 \\
\hline 28 & Tchèque & 24 & 5610 & 1570 & 4,3 \\
\hline 29 & Taïwanais & 29 & 5680 & 2910 & 5,1 \\
\hline 30 & Danois & 7 & 5250 & 325 & 1,3 \\
\hline Moyenne & & $\mathbf{1 0 1}$ & $\mathbf{3 7 2 8 0}$ & $\mathbf{2 1 6 9 6}$ & $\mathbf{3 , 6}$ \\
\hline
\end{tabular}

* Par ordre de population (langue maternelle) pour des communautés de 5000 de population et plus

Pour ce qui est du potentiel de demande pondéré, les quatre premiers rangs sont les mêmes que pour la langue maternelle (pendjabi, cantonnais, chinois n.d.a., mandarin). Elles sont suivies du coréen (qui passe du $8^{\mathrm{e}}$ langue maternelle au $5^{\mathrm{e}}$ rang langue parlée à la maison), de l'espagnol, du persan et du vietnamien. Les communautés allemande et française passent, elles, du $5^{\mathrm{e}}$ et du $7^{\mathrm{e}}$ rang, langue maternelle, au $13^{\mathrm{e}}$ et $10^{\mathrm{e}}$ rang, langue parlée à la maison, respectivement. On observe en fait que la plupart des communautés de souche européenne baissent de rang quand on passe de la statistique langue maternelle à la statistique langue parlée à la maison. Si on calcule l'indice vitalité linguistique, IVL= $\mathrm{I}_{2} / \mathrm{I}_{1}$, seulement 3 communautés de souche européenne sur 16 ont un IVL au-dessus de 0,5 (espagnol, russe, serbe) alors que pour les communautés de souche non-européenne (surtout asiatiques) c'est le contraire car seulement 3 sur 14 sont en dessous de 0,5 (tagalog, japonais, gujarati).

(c) Le potentiel de niveau d'offre $\left(I_{3}\right)$ soit le nombre de médecins par 1000 de population, langue maternelle), 2011 (communautés $>5000$ de population, langue maternelle)

Nous observons au Tableau 1 un potentiel de niveau d'offre moyen de 3,6 médecins par 1000 de population $\left(\mathrm{I}_{3}\right)$. Il y a quatre 
communautés linguistiques où ce potentiel est considérable (hindi 9,7; gujarati 9,9; français 11,9; ourdou 15,7). Dans 76,7 \% des cas, $\mathrm{I}_{3}$ varie entre 1 et 4 médecins par 1000 de population ce qui donne une valeur moyenne de 2,3 pour le sous-groupe excluant ces quatre communautés.

Une valeur de 2,3 par 1000 de population est proche des valeurs typiques de niveaux d'offre pour les systèmes des pays de l'OCDE (3,1; OCDE, 2013), des États-Unis (2,4; Globe and Mail, 2012), du Canada (2,4; Esmail, 2011) et des provinces du Canada (Colombie-Britannique, 2,49; Île-du-Prince-Édouard, 1,80; Nouvelle-Écosse, 2,40; Québec, 2,25;Esmail, 2011). Il semble donc que toutes attentes raisonnables soient gardées en ce qui a trait à la plupart des communautés linguistiques minoritaires de Colombie-Britannique.

Pour les communautés hindi, gujarati et ourdou (comme pour le français d'ailleurs) on observe de 4 à 7 fois plus de médecins per capita que pour la plupart des autres communautés. L'hindi est la langue nationale des Indes et les deux autres en sont des langues officielles. Comme ces trois minorités linguistiques indiennes sont relativement petites, ceci suggère la possibilité que ce résultat provienne d'un effet de retombée dû à l'importance démographique de la communauté pendjabi en ColombieBritannique. Celle-ci en est la plus grande minorité et elle comprenait 182920 locuteurs de langue maternelle en 2011. De plus, les médecins qui déclarent parler pendjabi en ColombieBritannique parlent aussi l'hindi (72\%), l'ourdou (40\%) et le gujarati $(10 \%)$. On observe donc un effet de retombée de services (spin-off) provenant de la communauté dominante pendjabi vers trois autres communautés minoritaires plus petites qui partagent une relation linguistique avec elle.

\section{B- Comparaisons avec le français et identification de caractéristiques structurantes uniques au français}

Le français vient de loin au premier rang en termes du potentiel d'offre de services médicaux avec 683 médecins parlant français en 2011 mais il ne vient qu'au $7^{\mathrm{e}}$ rang populationnel des langues maternelles minoritaires. Le français vient aussi au 
"D'autre part le français se démarque considérablement en ce qui a trait au niveau potentiel de services. »

"Ceci semble être une caractéristique structurante unique au français mais pas nécessairement. En effet trois communautés indiennes relativement petites partagent avec le français un niveau potentiel de services exceptionnel...» $10^{\mathrm{e}}$ populationnel rang des langues minoritaires parlées à la maison. Ceci suggère que la vitalité linguistique du français est relativement faible mais "typique » si on la considère par rapport à l'ensemble des autres langues de souche européenne. Le français se trouve à la médiane des valeurs soit sept langues qui ont une vitalité linguistique plus faible que le français (danois 0,062 ; néerlandais 0,097 ; ukrainien 0,111 ; allemand 0,157 ; italien 0,260 ; hongrois 0,265 ; tchèque 0,280 ) et huit langues qui en ont une plus forte (croate 0,325 ; grec 0,337 ; portugais 0,355 ; polonais 0,366 ; roumain 0,499 ; espagnol 0,505 ; russe 0,539 ; serbe 0,603 ). Dans l'ensemble il y a tendance pour les communautés de souche européenne de ne pas parler leur langue maternelle à la maison et d'être plus assimilées, ce qui résulterait en un amoindrissement relatif de la demande de services pour ces langues.

D'autre part le français se démarque considérablement en ce qui a trait au niveau potentiel de services. Si on le compare aux communautés minoritaires plus grandes que le français $(>50000$ individus, langue maternelle), le français fait bande à part par un facteur de 4 ou 5 (Figure 1).

Ceci semble être une caractéristique structurante unique au français mais pas nécessairement. En effet trois communautés indiennes relativement petites partagent avec le français un niveau potentiel de services exceptionnel (plus de 9 médecins par 1000 de population).

Nous avons suggéré plus haut que ces communautés indiennes bénéficieraient d'un mécanisme de retombée de services provenant d'une communauté dominante qui partage une relation linguistique avec elles. On pourrait invoquer un mécanisme semblable pour la CFASM de Colombie-Britannique mais il s'agit d'identifier une relation linguistique avec une communauté dominante. Nous proposons ici que les populations anglophones et allophones qui font souvent l'apprentissage du français dans un contexte canadien de dualité linguistique représentent cette communauté dominante qui crée un effet de retombée de services en français. En effet en 2006, 7 \% de la population de la Colombie-Britannique dont la langue maternelle n'est pas le français déclarait avoir une connaissance du français alors 
que la proportion de francophones n'était que de 1,7\%; il y aurait donc approximativement de 3 à 4 "francophiles " par francophone en Colombie-Britannique pour 2006 (Chavez et Bouchard-Coulombe, 2011) (FCFA, 2007) (Statistique Canada, 2012). Ceci suggère un potentiel de retombée linguistique considérable. Pour confirmer cette retombée linguistique parmi les rangs professionnels, soit pour les médecins de famille pratiquant en Colombie-Britannique, nous avons estimé la source d'apprentissage du français chez les médecins de famille pratiquant en Colombie-Britannique. Les résultats sont présentés au Tableau 2 pour 38 parmi 164 médecins de famille ayant gradué depuis 2000.

Figure $1-\mathrm{N}^{\circ}$ de médecins parlant la langue maternelle des sept plus grandes communautés linguistiques minoritaires de Colombie-Britannique (par 1000 de population)

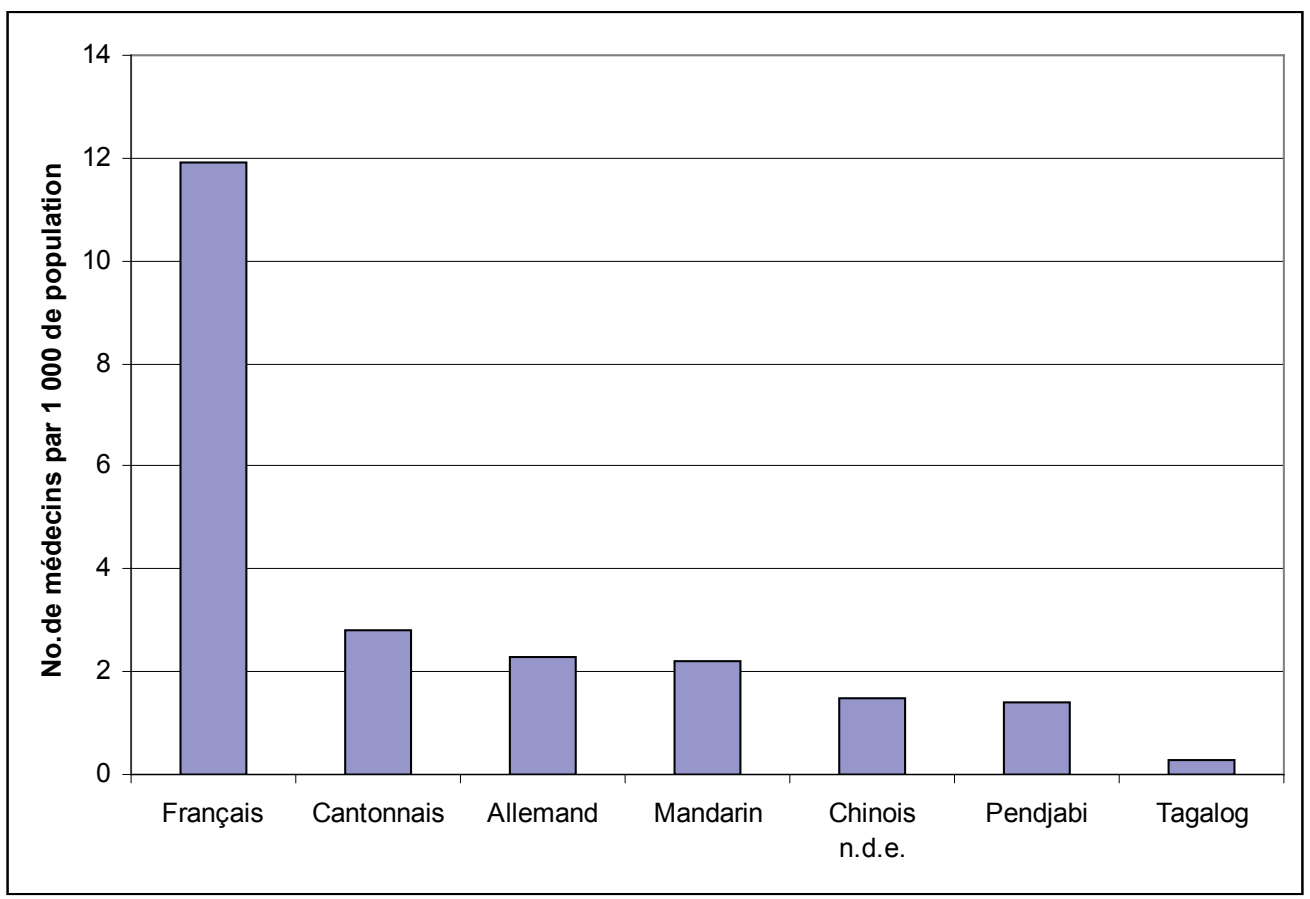


Tableau 2 - Apprentissage du français chez 38 médecins parlant français en Colombie-Britannique.

\begin{tabular}{|c|c|c|c|}
\hline Catégorie de réponse & $\begin{array}{c}\text { No. de } \\
\text { médecins }\end{array}$ & $\begin{array}{c}\text { \% de médecins } \\
\text { (catégories 1-5) }\end{array}$ & Université de formation \\
\hline Francophone de souche & 13 & 34,2 & $\begin{array}{c}\text { Laval(3), Montréal(2), Sherbrooke, } \\
\text { Alberta, Calgary, Dalhousie, } \\
\text { McGill, Ottawa(2), Western } \\
\text { Ontario }\end{array}$ \\
\hline $\begin{array}{c}\text { De programme scolaire } \\
\text { d'immersion française }\end{array}$ & 10 & 26,3 & $\begin{array}{c}\text { British Columbia(9), } \\
\text { Queen's(2), Ottawa, McMaster }\end{array}$ \\
\hline $\begin{array}{c}\text { De programme scolaire de } \\
\text { base }\end{array}$ & 3 & 7,9 & Bahrain, McGill(8), Montréal, \\
Ottawa, Liban
\end{tabular}

Ce tableau indique qu'il y $34,2 \%$ des médecins qui sont « de souche francophone " (dont 53,8 \% ont étudié la médecine en français ${ }^{2}$ ). Des $65,8 \%$ (près des deux tiers) qui sont « francophiles ", $48 \%$ tombe dans la catégorie "a vécu en milieu francophone" (dont 9 sur 25 ont étudié à une université « locale ») et $52 \%$ tombe dans la catégorie «a appris le français comme langue seconde en milieu scolaire anglophone " (tous ont étudié la médecine dans des universités anglophones sauf que cette information n'est pas disponible pour l'Université d'Ottawa). En sus, il est clair que l'Université McGill joue un rôle important pour la CFASM de la Colombie-Britannique car ses gradués représentent 21,1\% de l'échantillon. Comme la pratique d'une langue en milieu social ou à la maison est un facteur important qui favorise la bonne communication dans une langue seconde (Macintyre, Burns, et Jessome, 2011), cette université de Montréal qui travaille en milieu minoritaire anglophone est bien positionnée pour aider aussi le milieu francophone minoritaire en sensibilisant leurs étudiants aux besoins des communautés de langues officielles en situation minoritaire et en leur permettant d'enrichir leur capacité langagière en milieu de travail et en milieu social francophone. 
Finalement notons que plus du quart des médecins échantillonnés au Tableau 2 ont appris le français en immersion française et que ces derniers sont plus que trois fois plus nombreux que ceux qui ont appris le français en programme scolaire français de base. Les programmes d'immersion ne représentent que de 2 à $8,5 \%$ de la population d'élèves pour les années 2000 et 2013 respectivement (Allen 2004) (Statistique Canada, 2004) (Pope, 2013) alors que les programmes de base sont presque universels. Ces statistiques présentent un témoignage de l'impact concret que les programmes d'immersion française ont "sur le terrain ».

\section{Discussion}

"La conclusion

écrasante de notre étude est que les Anglophones et Allophones du Canada apprennent le français en très grand nombre ce qui résulte en un effet de retombée linguistique qui favorise l'offre de services en français pour la communauté francophone de ColombieBritannique. »

\section{Un mécanisme de retombée linguistique structurant}

La conclusion écrasante de notre étude est que les Anglophones et Allophones du Canada apprennent le français en très grand nombre ce qui résulte en un effet de retombée linguistique qui favorise l'offre de services en français pour la communauté francophone de Colombie-Britannique. Si cette hypothèse est correcte, il s'en suit un mécanisme structurant qui devrait aussi s'appliquer (a) aux autres professions de la santé et des services sociaux, (b) dans d'autres juridictions au travers du Canada, et (c) que le plus grand potentiel de services en français devrait se trouver là où les francophones sont plus minoritaires, une prédiction qui semble au premier abord contre-intuitive. Plus il y a d'Anglophones et d'Allophones qui peuvent apprendre le français à l'école par rapport au poids démographique d'une CFASM dans une juridiction provinciale ou territoriale (c.-à-d., plus le rapport de force démographique entre la communauté dominante et la communauté bénéficiaire est grand), plus l'effet de retombée de services serait grand. Deux études basées sur les données du recensement de 2006 confirment ces trois prédictions (Blaser, 2009) (Gauthier, Timony, et Wenghofer, 2012).

Selon ces études il y a en effet une grande représentation de professionnels et de professionnelles parlant français pour 
toutes les professions en comparaison du poids démographique des francophones, pour toutes les CFASM où Blaser (2009) fournit une taille de l'échantillon qui permet de faire de telles comparaisons.

Tableau 3 - Proportion de professionnels de la santé ayant une connaissance du français, par profession, et proportion de francophones dans ces provinces (Blaser, 2009 pour 2006; Gauthier, Timony, et Wenghofer, 2012 pour 2007†).

\begin{tabular}{|l|r|r|r|r|r|r|r|}
\hline & C.-B. & Saskatchewan & Alberta & N.-É. & Manitoba & Ontario & N.-B. \\
\hline Médecin & $19,6 \%$ & $12 \%$ & $14,9 \%$ & $20,3 \%$ & $15 \%$ & $\begin{array}{r}23 \% \\
15,8 \% \dagger\end{array}$ & $53,3 \%$ \\
\hline $\begin{array}{c}\text { Infirmiers et } \\
\text { infirmières }\end{array}$ & $6,7 \%$ & $4,6 \%$ & $7,3 \%$ & $9,5 \%$ & $8,5 \%$ & $11,8 \%$ & $48,8 \%$ \\
\hline Psychologues & $10,7 \%$ & $6,2 \%$ & $7,7 \%$ & $15 \%$ & $10,1 \%$ & $18,6 \%$ & $60,2 \%$ \\
\hline Autres professions & $8,1 \%$ & $5,2 \%$ & $7,9 \%$ & $11,8 \%$ & $10 \%$ & $13,3 \%$ & $50,8 \%$ \\
\hline$\%$ francophone & $1,5 \%$ & $1,6 \%$ & $1,9 \%$ & $3,6 \%$ & $3,8 \%$ & $4,5 \%$ & $32,7 \%$ \\
\hline
\end{tabular}

* Psychologues, travailleurs sociaux et travailleuses sociales

"...per capita de population francophone, le nombre de professionnels et de professionnelles de la santé et de services sociaux parlant français est plus grand lorsque le poids démographique d'une CFASM est plus faible. »
Le potentiel d'offre de services en français est exceptionnel pour toutes les CFASM et particulièrement pour les CFASM de l'Ouest du Canada. De plus les données de Blaser (2009) confirment que per capita de population francophone, le nombre de professionnels et de professionnelles de la santé et de services sociaux parlant français est plus grand lorsque le poids démographique d'une CFASM est plus faible. La Figure 3 illustre le nombre de professionnels et de professionnelles par 1000 de population francophone en fonction du poids démographique des francophones selon les données de Blaser (2009) présentées au Tableau 3. Nous avons converti les données par logarithme naturel pour tenir compte de la grande variabilité des résultats, dont le cas particulier du Nouveau-Brunswick qui se trouve à l'écart en axe des $\mathrm{X}$ en raison d'un poids démographique de francophones relativement grand dans cette province. 
Figure 2 - Logarithme du $\mathrm{n}^{\circ}$ de professionnels par 1000 de population francophone en fonction du logarithme du poids démographique de ces populations pour les 7 juridictions provinciales du Tableau 3. Cercles : Médecins; Triangles : psychologues, travailleurs sociaux, travailleuses sociales; Diamants : infirmiers et infirmières; Carrés : autres professions

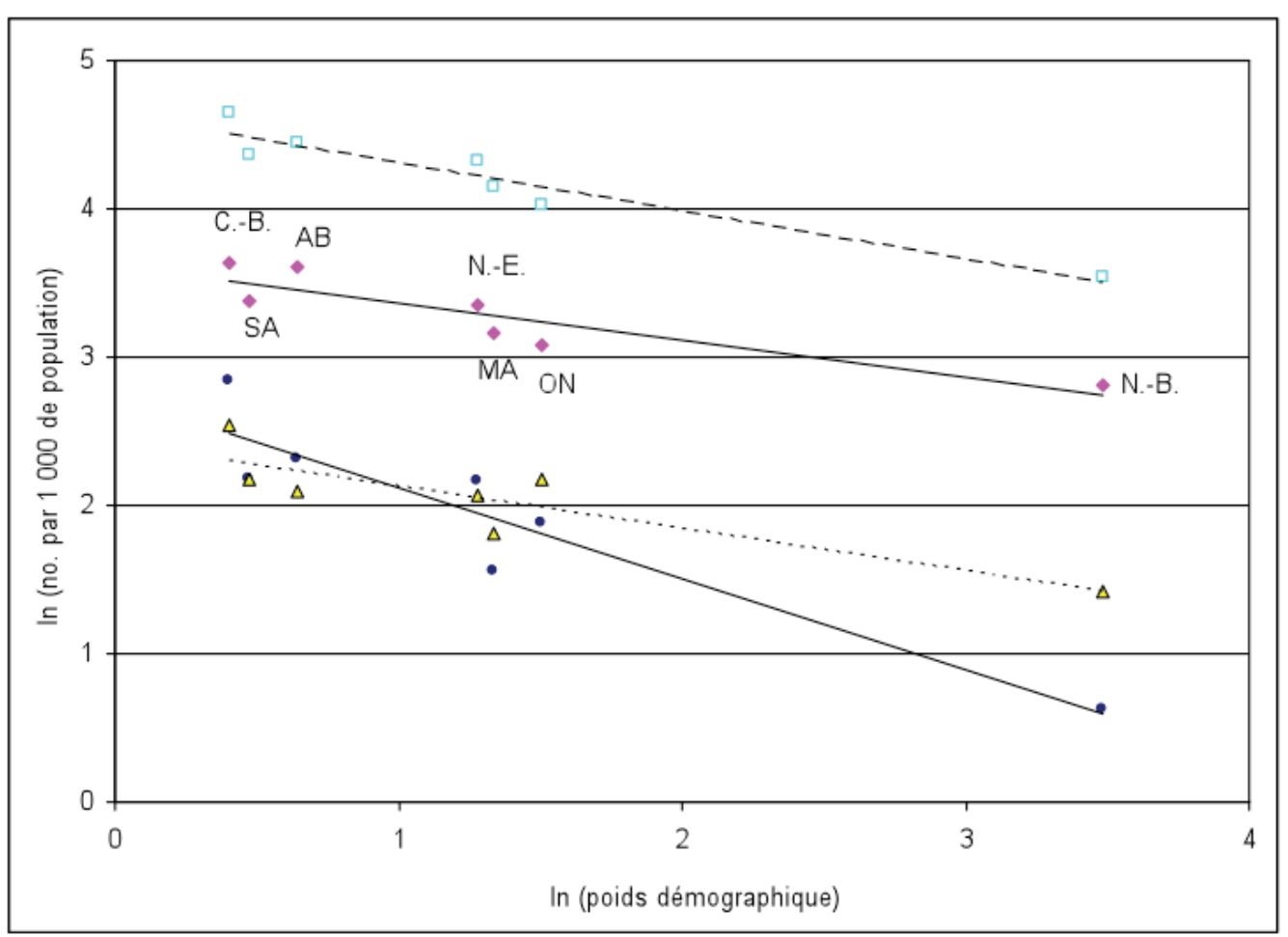


La transformation logarithmique produit des relations linéaires pour toutes les professions. Ceci démontre que le potentiel d'offre de services est plus grand plus une communauté francophone est minoritaire (et pour des raisons non-connues la pente est plus grande pour la profession médicale ce qui indique une tendance plus forte) et confirme la troisième prédiction issue de notre hypothèse. Ceci confirme aussi que les CFASM du Canada bénéficient d'un mécanisme de retombée linguistique structurant. Pour une CFASM favorisée comme celle de la ColombieBritannique, le défi principal des services en français n'est pas donc un défi de formation, de recrutement ou de rétention de professionnels et de professionnelles francophones (AFMC, 2006) (Statistique Canada, 2008) (CNFS, 2008; 2012) tel que le suggère le CCCFSM quand il affirme par exemple qu'il faudrait tripler, voire quadrupler le nombre d'inscription de francophones dans les programmes de formation en santé (CCCFSM, 2001, p. 28), ou qu'il affirme que le nombre de professionnels et de professionnelles parlant français est un enjeu qui continuera d'être une problématique clé de la santé de la population au cours des prochaines années (CCCFSM, 2007, p. 37). Le défi est plutôt celui de l'identification, de l'engagement, du recrutement, de la rétention et de la formation continue des professionnels et des professionnelles "francophiles » existants; ceux-ci représentent un potentiel latent de services sociaux et de santé en français qui peut surpasser de beaucoup le potentiel francophone.

\section{Un mécanisme de retombée linguistique unique}

Le mécanisme de retombée linguistique structurant identifié cidessus distingue la communauté francophone des autres grandes minorités linguistiques de Colombie-Britannique $(>25000$ de population) mais il existe aussi chez trois petites communautés indiennes. Le cas du français nous semble unique cependant de par son ampleur, d'abord parce que la communauté francophone de Colombie-Britannique est considérablement plus grande que ces trois dernières (Tableau 1) et ensuite parce que dans le cas des CFASM, il est soutenu par des programmes scolaires d'immersion 
"Le mécanisme de retombée linguistique structurant identifié ci-dessus distingue la communauté francophone des autres grandes minorités linguistiques de Colombie-Britannique (>25000 de population)..." française grandissants (Statistique Canada, 2004) (CPF, 2011) (Pope, 2013) qui « nourrissent » le bassin de professionnels parlant français partout au Canada (pour les communautés indiennes l'effet semble provenir d'un effort de recrutement ciblé). Depuis dix ans, les taux de participation annuels des programmes scolaires d'immersion française sont à la hausse partout au Canada, sauf au Nouveau-Brunswick. Pour l'ensemble du Canada $10 \%$ des élèves éligibles s'inscrivaient aux programmes d'immersion en l'an 2000 alors qu'en 2007 c'était 13,2\% et en 2011, 14 \% (CPF, 2011). Comme les élèves de programmes scolaires d'immersion française n'entrent sur le marché des services médicaux qu'une dizaine d'années après leur graduation (un effet de décalage), on peut prévoir que le nombre de professionnels et de professionnelles de la santé et des services sociaux parlant français continuera de croître en Colombie-Britannique et au Canada jusqu'en 2023, et probablement au-delà.

\section{L'enjeu de l'offre de services de santé en français en Colombie-Britannique : potentiel versus réalité}

Nous avons démontré qu'il existe un potentiel d'offre de services sociaux et de santé en français favorable en Colombie-Britannique. Toutefois les francophones y affirmaient récemment qu'il est difficile de trouver ces services et qu'approximativement $50 \%$

"L'enjeu principal pour les francophones de Colombie-

Britannique est donc de faire face à ce paradoxe et d'identifier les obstacles qui les empêchent d'exploiter un potentiel de service exceptionnel." d'entre eux n'en avaient pas trouvé (voir l'introduction). L'enjeu principal pour les francophones de Colombie-Britannique est donc de faire face à ce paradoxe et d'identifier les obstacles qui les empêchent d'exploiter un potentiel de service exceptionnel. Selon certaines des études nationales ou provenant d'autres provinces, la distribution relative des professionnels et professionnelles et de la population francophone pourrait être un obstacle (Blaser, 2009; de Moissac, et collab., 2012; Warnke, 2009; Warnke et Bouchard, 2013), ce que Gauthier,Timony et Wenghofer (2012) appellent une "mauvaise distribution». Selon de Moissac et collab. (2012) une mauvaise distribution peut être amplifiée lorsque l'on considère leur morcellement sur plusieurs professions. Ce scénario semble plausible pour les régions rurales de Colombie- 
Britannique mais pour la majorité des francophones vivant dans le Grand Vancouver et dans la région de la capitale provinciale, Victoria, où les services de santé et de services sociaux sont plus concentrés (Chavez et Bouchard-Coulombe, 2011), ce n'est pas le cas.

Un autre obstacle souvent mentionné est la validité de l'autoévaluation de la capacité langagière. D'une part les évidences de compétence langagière pour les étudiants issus de programmes scolaires en immersion française ont établi que leurs niveaux de compréhension écrite et orale sont comparables aux élèves francophones (CCA, 2007) (Genesee, 1978) (Genesee et Jared, 2008) (Lambert et Tucker, 1972). Du côté des allophones, $83 \%$ de ceux complétant des programmes scolaires d'immersion française indiquaient qu'ils étaient capables de converser en français, $66 \%$ indiquaient qu'ils avaient une bonne compréhension des nouvelles médiatiques, et $58 \%$ se sentaient capables de postuler pour du travail où la maitrise de la langue française est requise (CPF, 2011). D'autre part la compétence langagière en pratique professionnelle doit varier considérablement d'une profession à l'autre et elle n'est pas nécessairement étroitement liée au langage "technique " d'une profession puisque le client ne le connait pas. L'important c'est plutôt le langage d'accueil, un vocabulaire général pour décrire le système de santé, la langue sociale, et/ou la littératie de la santé et du mieux-être. Dans l'ensemble nous ne croyons pas que la validité de l'autoévaluation de la capacité langagière puisse changer nos résultats. Il y a un très haut niveau de médecins parlant français per capita de francophones en Colombie-Britannique car plus d'un tiers sont francophones de souche et le barème de compétence en pratique professionnelle n'est qu'un tiers plus bas que la capacité langagière de base.

Les obstacles qui nous semblent les plus difficiles à surmonter en Colombie-Britannique nous semblent donc être les suivants, par ordre d'importance croissante :

1) La disponibilité des professionnels et professionnelles Comme partout ailleurs au Canada, les listes d'attente pour des services en santé sont une réalité quotidienne pour les Franco-Colombiens. De plus, la plupart des médecins de famille 
"Ce problème est aigu car la plupart des parlant français ne portent pas de nom français en ColombieBritannique, pour les deux raisons suivantes : (a) Près des deux tiers sont des "francophiles" qui ne portent généralement pas un nom français. » n'acceptent pas de nouveaux clients (couramment $90 \%$ n'en acceptent pas selon le site Web de la BCCPS). Beaucoup de Britanno-Colombiens fréquentent donc des cliniques sans rendezvous (Global News, 2014) ce qui rend difficile l'appariement d'un médecin et de ses clients selon un critère linguistique, quelle que soit la communauté linguistique. Ce défi semble moins considérable cependant pour certaines professions comme pour les soins dentaires, les services pharmaceutiques, la médecine douce ou la médecine holistique, car ces services sont surtout offerts dans des milieux cliniques où la plupart des professionnels et des professionnelles acceptent des nouveaux clients.

\section{2) L'identification des parlant français parmi les profes- sionnels et les professionnelles}

Ce problème est aigu car la plupart des parlant français ne portent pas de nom français en Colombie-Britannique, pour les deux raisons suivantes : (a) Près des deux tiers sont des "francophiles " qui ne portent généralement pas un nom français. Par exemple, lorsque RésoSanté et le Collège Éducacentre ont offert l'atelier de formation "Soignez vos patients en français " (SVPF) en février 2013, sept participants étaient des étudiants inscrits au même cours de la Faculté de médecine de l'université de la Colombie-Britannique. Ils se connaissaient depuis au moins cinq mois mais tous ont été surpris de découvrir en arrivant à l'atelier qu'ils parlaient tous français; et (b) Le tiers des francophones de Colombie-Britannique sont de famille exogame (soit 87,8 \%; Chavez et Bouchard-Coulombe, 2011). Plusieurs ne portent donc pas un nom français de par leur association maritale ou de celle de leurs parents.

Pour contrecarrer ce phénomène, RésoSanté investit beaucoup d'efforts dans un outil de "Répertoire des professionnels et professionnelles parlant français » et un "Répertoire des médecins spécialistes " afin de faire valoir la richesse services disponibles en français. On trouve plus de 750 inscriptions dans chacun d'eux et la plupart portent des noms "non-français ». Le rôle de ces répertoires est de faire valoir cette richesse "cachée " et de sensibiliser et d'encourager la population francophone à « choisir les services en français " parmi une gamme de services (la plupart 
"L'attitude des anglophones en situation majoritaire joue donc un rôle déterminant dans la prestation de services en français. L'attitude de cette majorité reflète une logique d'équité selon laquelle on offre principalement les services en anglais, alors que pour les autres on offre un service succédané basé sur un service d'interprétation ... Cette approche nous semble fallacieuse parce qu'elle met toutes les communautés linguistiques sur le même pied sans tenir compte de la capacité d'offre pour ces communautés. » sont offerts dans des cliniques qui acceptent des nouveaux patients et où le défi de disponibilité mentionné ci-dessus n'existe pas).

\section{3) Les attitudes sociales qui influencent l'offre et la de- mande de services en français}

Nous avons mentionné en introduction qu'un projet d'offre de services en français dans une clinique de Vancouver avait été discontinué parce qu'il avait été perçu comme inéquitable par rapport à d'autres minorités linguistiques qui sont démographiquement plus importantes. Cette observation concorde avec les constats de l'étude de Forgues, Bahi et Michaud (2011) sur les facteurs sociaux qui influencent l'offre de services en français dans quatre hôpitaux du Manitoba, de l'Ontario, du Nouveau-Brunswick et de la Nouvelle-Écosse. Ces auteurs remarquent que du côté des anglophones on n'est pas enclin à reconnaitre le droit des francophones de recevoir des services en français, surtout pour les francophones qui sont bilingues pour les raisons suivantes :

On comprend mal comment, dans un contexte multiculturel, on fait plus d'efforts pour les francophones que pour les autres minorités ethniques. On met ainsi sur le même pied d'égalité le droit des immigrants et celui des francophones, oubliant l'existence de la Loi sur les langues officielles. Le droit des patients de recevoir des services en français se définit dans une perspective de gestion : la langue de communication ayant une influence certaine sur la qualité des soins offerts, il n'y a pas de différence entre un patient francophone et un nouvel arrivant qui parle une autre langue que l'anglais. p. 13

L'attitude des anglophones en situation majoritaire joue donc un rôle déterminant dans la prestation de services en français. L'attitude de cette majorité reflète une logique d'équité selon laquelle on offre principalement les services en anglais, alors que pour les autres on offre un service succédané basé sur un service d'interprétation (il y a des retards et des coûts additionnels 
pour les services d'interprétation). Cette approche nous semble fallacieuse parce qu'elle met toutes les communautés linguistiques sur le même pied sans tenir compte de la capacité d'offre pour ces communautés. La "logique d'équité » d'une perspective de gestion qui met l'accent sur une approche interprétative basée strictement sur la demande ignore l'offre active de services pour une communauté où la capacité de service existe déjà et par conséquent handicape cette communauté; ceci nous semble fondamentalement non-équitable. Ceci rappelle l'utopie présentée par KurtVonnegut Jr. dans son essai " Harrison Bergeron » (1961) où l'état s'assure que chaque américain est égal par le truchement d'un fonctionnaire, le "Handicapper General ", qui impose à chaque citoyen un fardeau qui est proportionnel à son talent, pour contrecarrer ce talent (par ex., des poids pour les danseurs de ballet). Selon cette logique il faudrait ajuster les services aux Anglophones majoritaires en les forçant à recevoir leur services par l'entremise de médecins non-anglophones, afin qu'ils aient accès au même niveau de service linguistique que les communautés minoritaires, ce qui est absurde ! Il nous semble qu'une logique d'équité qui tiendrait compte tant de l'offre que de la demande est plus équitable car elle obtiendrait sûrement de meilleurs résultats pour les communautés mieux nanties tout en permettant d'économiser des ressources qui pourraient être mises à la disposition d'autres communautés moins bien nanties. Cette approche pragmatique serait gagnante-gagnante et ne serait pas nécessairement dispendieuse. Un leadership qui

"Un leadership qui propose une politique d'offre active de services dans la langue du client auprès des professionnels, des professionnelles et de la communauté,..." propose une politique d'offre active de services dans la langue du client auprès des professionnels, des professionnelles et de la communauté, partout où cela est possible, pourrait obtenir des succès « immédiats » sans frais onéreux (dans les cliniques privées par exemple) et permettrait d'habiliter les gestionnaires en santé à offrir, dans la mesure du possible, des services linguistiquement appropriés et de meilleure qualité.

Nous illustrons une telle approche de façon caricaturale pour les 30 communautés linguistiques du Tableau 2 en présentant les indicateurs du potentiel d'offre et de demande $\mathrm{I}_{1}$ et $\mathrm{I}_{2}$ regroupés en trois selon un rang grandissant (Tableau 4). 
Tableau 4 - Correspondance du potentiel de demande et d'offre de services dans la langue des 30 plus grandes communautés linguistiques de C.-B en 2011*. Le rang du niveau d'offre individuel est donné entre parenthèses.

\begin{tabular}{|c|c|c|c|c|}
\hline & \multicolumn{4}{|c|}{ Niveau d'offre de service $\left(I_{3}\right)$} \\
\hline & Rangs & $21-30$ & $11-20$ & $1-10$ \\
\hline \multirow{3}{*}{$\begin{array}{c}\text { Demande } \\
\text { élevée } \\
\left(\mathrm{I}_{2}\right) \\
\text { Demande } \\
\text { basse }\end{array}$} & $1-10$ & $\begin{array}{c}\text { pendjabi (23) } \\
\text { persan (27) } \\
\text { coréen (29) } \\
\text { tagalog (30) }\end{array}$ & $\begin{array}{c}\text { cantonnais (11) } \\
\text { mandarin (17) } \\
\text { allemand (15) }\end{array}$ & $\begin{array}{l}\text { français (2) } \\
\text { espagnol(9) }\end{array}$ \\
\hline & $11-20$ & $\begin{array}{c}\text { italien (21) } \\
\text { vietnamien (25) } \\
\text { portugais (26) } \\
\text { japonais (28) }\end{array}$ & $\begin{array}{c}\text { russe (13) } \\
\text { ukrainien (16) } \\
\text { néerlandais (19) }\end{array}$ & $\begin{array}{c}\text { hindi (4) } \\
\text { arabe (5) } \\
\text { polonais (8) }\end{array}$ \\
\hline & $21-30$ & danois (24) & $\begin{array}{c}\text { serbe }(12) \\
\text { hongrois (14) } \\
\text { grec }(18) \\
\text { croate }(20)\end{array}$ & $\begin{array}{c}\text { ourdou (1) } \\
\text { gujarati (3) } \\
\text { taïwanais (6) } \\
\text { tchèque (7) } \\
\text { roumain (10) }\end{array}$ \\
\hline
\end{tabular}

^ Chinois n.d.a (22) est exclu car c'est un groupe de langues (un ensemble de plusieurs langues ou dialectes)

Ce tableau suggère par exemple que les communautés francophones et espagnoles sont relativement favorisées tant pour l'offre que pour la demande et qu'une approche d'offre active permettrait de réaliser une plus grande qualité et une plus grande efficacité de services pour ces communautés.

Cette représentation caricaturale suggérerait aussi des interventions suivantes de type suivant :

- Cliniques spécialisées pour les communautés où la demande est grande mais l'offre de services est faible (pendjabi, persan, coréen, tagalog), surtout si une de ces communautés se retrouve surtout dans une zone géographique définie. L'interprétation deviendrait alors un service complémentaire en dehors des zones géographiques favorables ou en dehors d'heures de service privilégiées. 
- Efforts de recrutement là où la demande est grande et l'offre modérée (mandarin, cantonnais, allemand);

- Services d'interprétation si la demande et l'offre sont modérées ou faibles.

Nous laissons soin au lecteur d'imaginer d'autres scénarios.

\section{Conclusion}

"...le nombre de professionnels et professionnelles parlant français en Colombie-Britannique est considérable et continuera de l'être en raison d'un effet de retombée linguistique provenant des communautés dominantes anglophone et allophones qui apprennent le français en grand nombre. "
Bien que les évidences présentées en introduction indiquent qu'il existe une pénurie de services médicaux en français en ColombieBritannique et ailleurs au Canada, les évidences présentées ici indiquent que le nombre de professionnels et professionnelles parlant français en Colombie-Britannique est considérable et continuera de l'être en raison d'un effet de retombée linguistique provenant des communautés dominantes anglophone et allophones qui apprennent le français en grand nombre. L'enjeu des services en français en Colombie-Britannique n'est donc pas celui de la formation, du recrutement ou de la rétention des professionnels et des professionnelles francophones tel que le suggère le CCCFSM (2001; 2007). L'enjeu est celui des attitudes sociales mitigées qui prévalent autour de l'offre et la demande de services en français et celui de la disponibilité, de l'identification et de l'outillage des professionnels et des professionnelles francophones et francophiles existants. Il nous semble donc essentiel que les CFASM du Canada et leurs réseaux de santé et de services sociaux entament l'élaboration d'un argumentaire novateur qui propose une logique d'équité et un leadership qui tiennent compte tant de l'offre que de la demande dans l'élaboration de politiques de services et de programmes de santé et de services sociaux dans la langue du client.

\section{Notes}

C'est le $7^{\mathrm{e}}$ rang en Alberta, le $5^{\mathrm{e}}$ en Saskatchewan, les $4^{\mathrm{es}}$ aux Territoires-du-Nord-Ouest et au Manitoba; le $3^{\mathrm{e}}$ au Terre-Neuve et Labrador, le $2^{\mathrm{e}}$ au Nunavut et le $1^{\mathrm{er}}$ rang dans les cinq autres CFASM.

2 Comme l'Université d'Ottawa a des volets anglophone et francophone, nous en avons alloué un pour chacun. 


\section{Reconnaissance}

Cette initiative est financée par Santé Canada. Les opinions exprimées ici ne reflètent pas nécessairement celles de Santé Canada et de la Société Santé en français.

\section{Bibliographie}

AFMC (2006). Des médecins et des soins de qualité pour les communautés francophones minoritaires du Canada, [Rapport final sur les activités et les résultats du projet déposé à Santé Canada], Ottawa, Association des facultés de médecine du Canada, $37 \mathrm{p}$.

ALEKSYNSKA, Mariya, et Yann ALGAN (2010). Assimilation and Integration of Immigrants in Europe, [Discussion Paper No. 5185], Bonn, Institute for the Study of Labor, 47 p.

ALLEN, Mary (2004). «Reading achievement of students in French immersion programs », Educational Quarterly Review, Vol. 9, No 4, p. 25-30.

BEAULIEU, Marielle (août 2010). Portrait des besoins des professionnels de la santé : Inventaire des programmes de formation linguistique et d'adaptation culturelle, perceptions des gestionnaires d'établissements de santé, Province de la Colombie-Britannique, [rapport final], Ottawa, Société Santé en français, 55 p.

BLASER, Christine (avril 2009). Professionnels de la santé et minorités de langue officielle au Canada, [Recensement 2001 et 2006], Ottawa, Statistique Canada, 62 p.

CCCFSM (2001). Pour un meilleur accès à des services de santé en français, [rapport au Ministre fédéral de la santé], Ottawa, Comité consultatif des communautés francophones en situation minoritaire, $54 \mathrm{p}$.

CCCFSM (2007). Pour un nouveau leadership en matière d'amélioration des services de santé en français, [rapport au Ministre fédéral de la santé], Ottawa, Comité consultatif des communautés francophones en situation minoritaire, $58 \mathrm{p}$.

CHARTRAND, Michel (mai 2010). "En 2006, on comptait 400000 francophones anglicisés de plus : L'assimilation fait la différence de poids ", L'aut'Journal, No 289, réf. du $1^{\text {er }}$ mai 2013, http:// archives.lautjournal.info/autjourarchives.asp? article $=3779 \&$ noj $=289$

CHAVEZ, Brigitte, et Camille BOUCHARD-COULOMBE (2011). Portrait des minorités de langue officielle au Canada : les francophones de la Colombie-Britannique, [rapport], Ottawa, Statistique Canada, $80 \mathrm{p}$.

CHISWICK, Barry R. (1991). « Reading, Speaking, and Earnings Among Low-Skilled Immigrants », Journal of Labor Economics, Vol. 9, p. 149-170.

CNFS (2008). Un projet de formation qui a fait et continue de faire ses preuves - La phase II du CNFS 2003-2008 : cinq années déterminantes, [rapport], Ottawa, Consortium national de formation en santé, $14 \mathrm{p}$.

CNFS (2012). Cadre de référence pour la formation à l'offre active des services de santé en français, Ottawa, Consortium national de formation en santé, $27 \mathrm{p}$.

CCA (mai 2007). Lessons in learning : French-immersion education in Canada, [rapport], Ottawa, Conseil canadien sur l'apprentissage, $13 \mathrm{p}$.

CPF (2011). Annual FSL Enrolment in Canada 2006-2001 : National Summary Statistics, Ottawa, Canadian Parents for French, réf. du 18 février 2014, http://cpf.ca/en/research-advocacy/ research/enrolmenttrends/ 
DE MOISSAC, Danielle, et collab. (2012). Disponibilité et accessibilité des services de santé en français au Manitoba, [rapport final], Université de St-Boniface/Institut franco-ontarien de l'Université Larentienne, $90 \mathrm{p}$.

DUSTMANN, Christian (1994). «Speaking Fluency,Writing Fluency, and Earnings of Immigrants », Journal of Population Economics. Vol. 7, p. 133-156.

ESMAIL, Nadeem (2011). «Les effectifs médicaux au Canada », Toronto, Institut Fraser, Perspectives, Vol. 4, No 3, p 6-13.

FCFA (2007). Profil de la communauté francophone de la Colombie-Britannique, [rapport, Ottawa, Fédération des communautés francophones et acadienne du Canada, $24 \mathrm{p}$.

FORGUES, Éric, Boniface BAHI et Jacques MICHAUD (2011). L'Offre de services en français en contexte francophone minoritaire, [rapport], Ottawa, Institut canadien de recherche sur les minorités linguistiques, $182 \mathrm{p}$.

FOR GUES, Éric, et Rodrigue LANDRY (2006). Définitions de la francophonie en situation minoritaire : Analyse de différentes définitions statistiques et de leurs conséquences, [rapport], Ottawa, Consortium national de formation en santé et Société santé en français, $22 \mathrm{p}$.

FOR GUES, Éric, Rodrigue LANDRY et Jonathan BOUDREAU (2009). Qui sont les francophones? Analyse de définitions selon les variables du recensement, [rapport], Ottawa, Consortium national de formation en santé, $40 \mathrm{p}$.

GABRIEL, Patricia (2012). Physicians launch innovative pilot project to improve care for patients who don't speak English, réf. du 5 juin 2014, https://www.divisionsbc.ca/provincial/FNWinterpretation

GAGNON-ARPIN, Isabelle (2011). Perceived Health and Access to Care in Minority Settings :The Case for Canada's Official Language Minorities, Ottawa, Lambert Academic Publishing, 160 p.

GAUTHIER, Alain P., Patrick E.TIMONY et Elizabeth F.WENGHOFER (décembre 2012). «Sur la distribution géographique des médecins francophones de l'Ontario ", Can. Fam. Physician, Vol. 58, p. 717-724.

GENESEE, Fred (1978). «A longitudinal evaluation of an early immersion school program », Canadian Journal of Education, Vol. 3, p. 31-50.

GENESEE, Fred, et Debra JARED (2008). «Literacy Development in Early French Immersion Programs ", Canadian Psychology, Vol. 49, № 2, p. 140-147.

GIGUÈRE, Louis (2013). «Les services de santé en français pour les communautés francophones et acadiennes en situation minoritaire du Canada : bonification du schéma du CCCSFM ", New Perspectives in Social Sciences, Vol. 9, No 1, p. 319-345.

GIGUÈRE, Louis (2014). «Validation d'un schème national sur développement des services de santé pour les communautés francophones et acadiennes en situation minoritaire au Canada : arrimage à des assises théoriques et modélisation ", New Perspectives in Social Sciences,Vol. 9, № 2, p. 207-236.

GLOBAL NEWS (14 janvier 2014). Family Doctors Difficult to Find Throughout Canada, réf. du 17 mars 2014, http://globalnews.ca/video/1062269/family-doctors-difficult-to-find-throughout-canada

GLOBE AND MAIL (15 novembre 2012). Canada boasts record number of doctors, but is it enough?, réf. du 18 février 2014, http://www.theglobeandmail.com/life/health-and-fitness/health/canadaboasts-record-number-of-doctors-but-is-it-enough/article5340309/

IRFA (2012). Le déclin du français au Canada, Institut de recherche sur le français en Amérique, réf. du $1^{\text {er }}$ mai 2013, http://www.irfa.ca/site/wp-content/uploads/2012/07/graphique-dinformation.pdf 
Lacroix, Frédéric, et Patrick Sabourin (2006). Les services de santé au Canada : Une perspective linguistique, L'Action nationale, réf. du 9 octobre 2012, http://www.actionnationale.qc.ca/index. php?option=com_contentettask $=$ viewetid $=129$ etItemid $=99999999$

LAMBERT,Wallace E., et G. R.TUCKER (1972). The Bilingual Education of Children :The St. Lambert Experiment, Rowley, Massachusetts, Newsbury House, 248 p.

LANDRY, Rodrigue (2010). Petite enfance et autonomie culturelle : Là où le nombre le justifie...V, [rapport], Moncton, Institut canadien de recherche sur les minorités linguistiques, $102 \mathrm{p}$.

LENTZ, François (2004). Apprendre le/en français en milieu minoritaire : Quelques enjeux didactiques sur la langue, Québec, [Actes du 9e Colloque de l'AIRDF], p. 1-14.

MACINTYRE, Peter D., Carolyn BURNS et Alison JESSOME (2011). "Ambivalence of communicating in a second language: A qualitative study of French immersion students' willingness to communicate", The Modern Language Journal, 95(1), p. 94.

MINISTÈRE DE LA JUSTICE (1985). Loi sur les langues officielles, L.R.C. (1985), ch. 31 (4e suppl.), réf. du 9 octobre 2012, http://laws.justice.gc.ca/fra/lois/O-3.01/TexteComplet.html

MINISTÈRE DE LA JUSTICE (2005). Loi modifiant la Loi sur les langues officielles (promotion du français et de l'anglais), L.C. 2005, ch. 41, réf. du 9 octobre 2012, http://laws.justice.gc.ca/fra/ LoisAnnuelles/2005_41/page-1.html

NATIONAL POST (8 mai 2013).Young, suburban and mostly Asian : Canada's immigrant population surges, National Post, réf. du 12 juin 2014, http://news.nationalpost.com/2013/05/08/youngsuburban-and-mostly-asian-canadas-immigrant-population-surges/

OCDE (2013). Panorama de la santé 2013 : Les indicateurs de l'OCDE, réf. du 12 février 2014, http://dx.doi.org/10.1787/health_glance-2013-fr

PATRIMOINE CANADIEN (2007). Guide à l'intention des institutions fédérales : Loi sur les langues officielles - Partie VII, Ottawa, $84 \mathrm{p}$.

PLS (2014). En santé en C.-B., 2013-2014 Highlights, Provincial Language Service, réf. Du 12 juin 2014, http://www.phsa.ca/NR/rdonlyres/624B39F0-2DE6-4B02-A160-15C7A34B839D/6 9765/20132014FSHighlightsFinalEN.pdf.

POCOCK, Joanne, et collab. (2011). Rapport d'accompagnement des Profils socio-économiques des collectivités anglophones du Québec par territoire des CSSS et des RSS, [rapport], Ottawa, Agence de la santé publique du Canada, $135 \mathrm{p}$.

POPE, Danielle (2013). «French immersion student numbers up across B.C. », Victoria News, réf. du 12 février 2014, http://www.vicnews.com/news/223375361.html

RÉSOSANTÉ (2005). Évaluation des besoins : Services de santé en français à la disposition de la population francophone des Tri-Cities, [rapport final du projet],Vancouver, RésoSanté Colombie-Britannique, $76 \mathrm{p}$.

RÉSOSANTÉ (2006). Préparer le terrain en Colombie-Britannique, [rapport final], Vancouver, RésoSanté Colombie-Britannique, $92 \mathrm{p}$.

SANTÉ CANADA (2008). Aperçu du Coût de Formation des Professionnels de la Santé, [rapport], Ottawa, $40 \mathrm{p}$.

STATISTIQUE CANADA (2004). Education Matters : Immersion 30 Years Later, réf. du 12 juin 2014, http://www.statcan.gc.ca/pub/81-004-x/200406/6923-eng.htm 
STATISTIQUE CANADA (2012). Le français et la francophonie du Canada : Langue, Recensement de la population de 2011, réf. du 12 juin 2014, http://www12.statcan.gc.ca/census-recensement/2011/as-sa/98-314-x/98-314-x2011003_1fra.cfm

VONNEGUT, Kurt Jr. (1961). Harrison Bergeron, réf. du 17 mars, 2014, https://archive.org/stream/ HarrisonBergeron/Harrison\%20Bergeron_djvu.txt

WARNKE, Jan (6 novembre 2009). Professionnels de la santé et capacité en langue officielle minoritaire : dimensions contextuelles de l'accès à la santé, Ottawa, [Colloque scientifique], La Santé des communautés de langue officielle en situation minoritaire du Canada, Santé Canada, 19 p.

WARNKE, Jan, et Louise BOUCHARD (2013). «Validation de l'équité d'accès des CLOSM aux professionnels de la santé dans les régions sociosanitaires du Canada ", Canadian Journal of Public Health 104 (6) Supplément 1 : S49 - S59.

ZAR, Jerrold H. (1984). Biostatistical Analysis, $2^{e}$ édition, Prentice Hall, New-Jersey, 718 p. 


\section{ANNEXE 1}

Le nombre de médecins parlant la langue de communautés en situation minoritaire en Colombie-Britannique, de 2009 à 2014

\begin{tabular}{|c|c|c|c|c|c|c|}
\hline Rang* $^{*}$ & Communauté & $\mathbf{2 0 0 9}$ & $\mathbf{2 0 1 0}$ & $\mathbf{2 0 1 1}$ & $\mathbf{2 0 1 2}$ & $\mathbf{2 0 1 4}$ \\
\hline $\mathbf{1}$ & Pendjabi & 170 & 239 & 249 & 274 & 289 \\
\hline $\mathbf{2}$ & Cantonnais & 213 & 366 & 370 & 395 & 376 \\
\hline $\mathbf{3}$ & Chinois n.d.a. $\dagger$ & 323 & 193 & 183 & 192 & 310 \\
\hline $\mathbf{4}$ & Mandarin & 120 & 191 & 207 & 225 & 261 \\
\hline $\mathbf{5}$ & Allemand & 320 & 177 & 169 & 172 & 245 \\
\hline $\mathbf{6}$ & Tagalog & 20 & 20 & 17 & 19 & 20 \\
\hline $\mathbf{7}$ & Français & 998 & 725 & 683 & 695 & 1161 \\
\hline $\mathbf{8}$ & Coréen & 26 & 24 & 21 & 24 & 32 \\
\hline $\mathbf{9}$ & Espagnol & 214 & 158 & 148 & 164 & 229 \\
\hline $\mathbf{1 0}$ & Persan & 39 & 18 & 18 & 19 & 52 \\
\hline $\mathbf{1 1}$ & Vietnamien & 21 & 20 & 24 & 22 & 21 \\
\hline $\mathbf{1 2}$ & Hindi & 194 & 241 & 240 & 283 & 304 \\
\hline $\mathbf{1 3}$ & Italien & 73 & 52 & 45 & 41 & 55 \\
\hline $\mathbf{1 4}$ & Néerlandais & 71 & 54 & 48 & 49 & 61 \\
\hline $\mathbf{1 5}$ & Russe & 89 & 69 & 60 & 68 & 68 \\
\hline $\mathbf{1 6}$ & Japonais & 21 & 9 & 9 & 10 & 26 \\
\hline $\mathbf{1 7}$ & Polonais & 91 & 73 & 66 & 73 & 84 \\
\hline $\mathbf{1 8}$ & Portugais & 19 & 14 & 15 & 14 & 21 \\
\hline $\mathbf{1 9}$ & Arabe & 75 & 85 & 82 & 88 & 87 \\
\hline
\end{tabular}

* Par ordre de population (langue maternelle) pour des communautés de 10000 de population et plus

† Chinois non déclaré ailleurs, c.-à-d., excluant le mandarin, le cantonnais, le hakka, le taïwanais, le chaochow, le fou-kien et le shanghaïen. Ce groupe représente un ensemble de plusieurs langues ou dialectes, pas une communauté linguistique cohérente 